\title{
Thematische Karten als Grundlage der ländlichen Entwicklung in Äthiopien
}

\section{Einleitung}

Äthiopien ist für die Weltöffentlichkeit das Land der Hungersnöte, der Umweltzerstörung und der Bürgerkriege. Nur wenige sind sich bewußt, wie riesig, agro-ökologisch differenziert und kulturell divergent dieses Gebirgsland am Horn von Afrika in Wirklichkeit ist. Dieser mangelhafte Informationsstand ist nicht nur bei Außenstehenden, sondern auch unter Experten und lokalen Institutionen weitverbreitet.
Eines der wichtigsten, für uns längst selbstverständlichen Hilfsmittel zur Erfassung der Verteilung, des Zustandes und der Dynamik von natürlichen Ressourcen, Landnutzungs-Systemen und Verteilungsmustern sowie von Humanfaktoren sind thematische Karten. Realitätsnahe Angaben über menschliche Aktivitäten im Kontext lokaler Umweltparameter sowie zur Entwicklung möglicher Szenarien für die zukünftige Entwicklung eines Raumes benötigen eine solide topographische Basis zur Darstel-

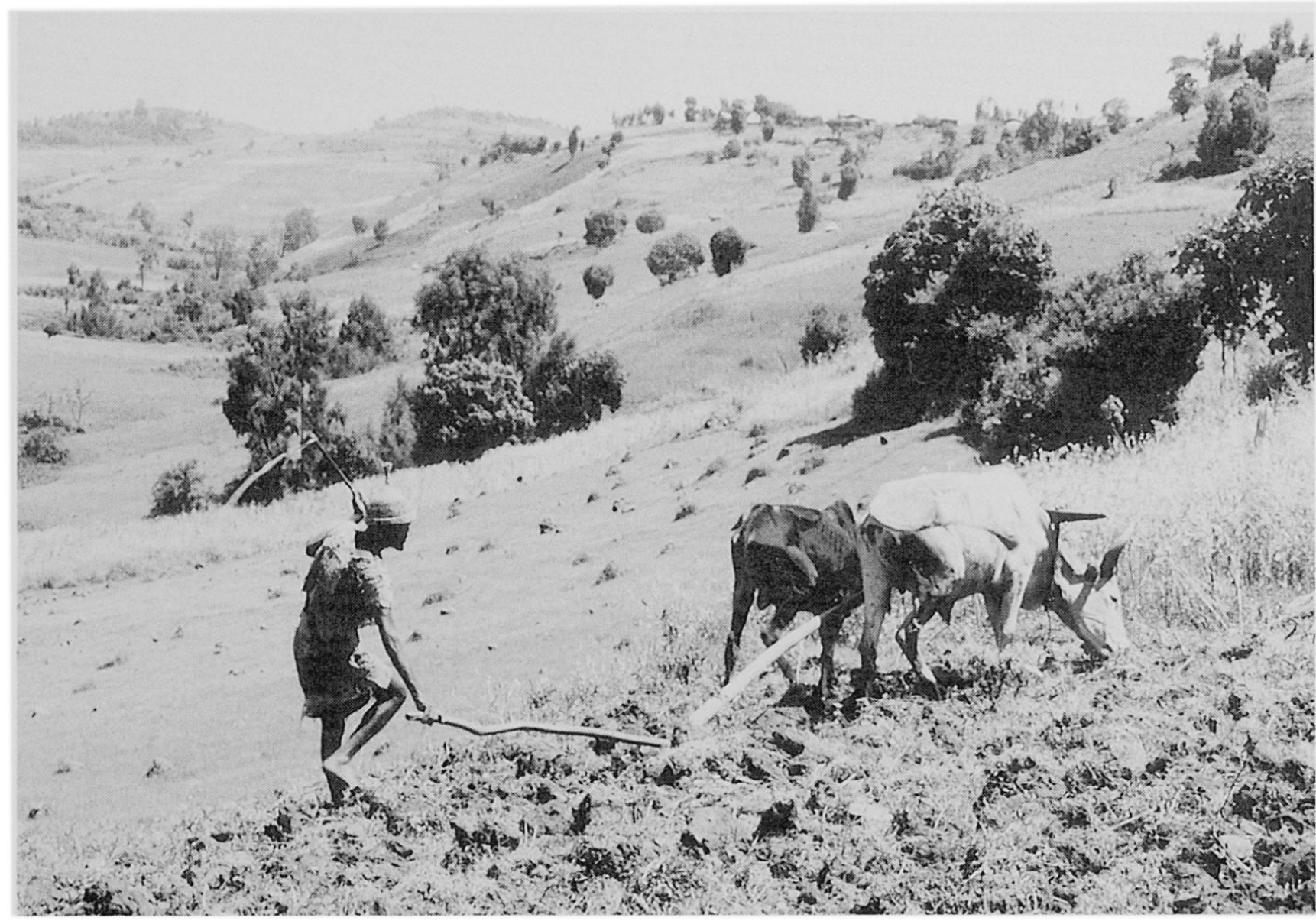

Abb. 1 Äthiopischer Selbstversorgungsbauer mit Ochsenpflug in einer stark genutzten Landschaft. Trotz verhältnismäßig hoher Produktivität sind die Degradationsprozesse durch Bodenerosion äußerst alarmierend. H. Hurni, Station Anjeni, Juni 1985. 
lung räumlicher Information. Das Ziel dieses Artikels ist es, die Hauptprobleme Äthiopiens kurz aufzuzeigen und die Rolle topographischer und thematischer Karten im Entwicklungsproze $B$ dieses ärmsten Landes der Erde mit einem Bruttosozialprodukt pro Kopf der Bevölkerung von knapp 100 US\$ (1986) zu unterstreichen.

In Äthiopien leben fast 50 Millionen Menschen und 70 Millionen Stück Vieh, d. h. ähnliche Zahlengrößen wie in der restlichen, achtmal größeren Sahelzone zusammen. Die Ursachen dieser Konzentration sind leicht erklärbar: Es sind die Höhenstufen oberhalb $1300 \mathrm{~m}$ bis $3600 \mathrm{~m}$ ü. M., die rund $43 \%$ des 1,221 Millionen Quadratkilometer großen Landes ausmachen und trotz der Sahel-Breitengrade von $6^{\circ}-19^{\circ} \mathrm{n}$. Br. mit durchschnittlich 500-2000 mm Jahresniederschlägen und gemäßigten bis kühlen Temperaturen optimale Bedingungen für Regenfeldbau und Viehwirtschaft aufweisen. So leben $80 \%$ der Bevölkerung und $60 \%$ des Viehs im Gebirge (CONSTABLE, 1985), die Leute als seßhafte Bauern in einer Subsistenzlandwirtschaft, basierend auf Getreidebau, Ochsenpflug-Systemen und einer minimalen marktwirtschaftlichen Abhängigkeit. Rund 85\% der Bevölkerung sind in der Landwirtschaft tätig und fast alle davon als Selbstversorger(innen).

\section{Ressourcennutzung, Hungersnöte und ländliche Entwicklung}

Vier Faktoren begründen die hohe Anfälligkeit der äthiopischen Bauern für Hungersnöte: Zum einen besteht nach Mesfin Wolde-Mariam (1984) mit der Selbstversorgungs-Landwirtschaft eine hohe Unabhängigkeit der Bauern von staatlichen und marktwirtschaftlichen Systemen, was bewirkt, daß ein Transportsystem und entsprechende Lagerkapazitäten für Getreide kaum vorhanden sind, also in Notzeiten auch nicht als Netzwerke zur Versorgung der ländlichen Bevölkerung mit Hilfsgütern funktionieren können. Zum zweiten ist die Sahel-Breitenzone auch in Äthiopien anfällig für markante Niederschlags-Variabilitäten, was wegen der hohen Bevölkerungsdichten bei einem regionalen Produktionsausfall, z. B. durch Dürre oder andere Faktoren, immer in eine Krisensituation mündet, verstärkt je nach Region durch Bürgerkriege, hohe Unzugänglichkeit oder große historische Umweltschädigung. Zum dritten schließlich ist die letztgenannte Degradation der natürlichen Ressourcen Vegetation, Boden und Wasser in vielen, vor allem nördlichen Gebieten Äthiopiens schon so weit fortgeschritten, $\mathrm{da} ß$ die Bedingungen für einen Selbstversorger bedeutend schlechter sind als zu Beginn landwirtschaftlicher Aktivitäten vor einigen Jahrtausenden. Die Produktivität der Böden ist oft auf unter $50 \%$ ihres ursprünglichen Niveaus gesunken. Zum vier- ten schließlich hat die große Bevölkerungszunahme von $2,9 \%$ pro Jahr in diesem Jahrhundert wesentlich zu einer Verstärkung der Anfälligkeit für Hungersnöte geführt, da heute in einer betroffenen Region meist doppelt so viele Leute wohnen als noch vor 20 Jahren.

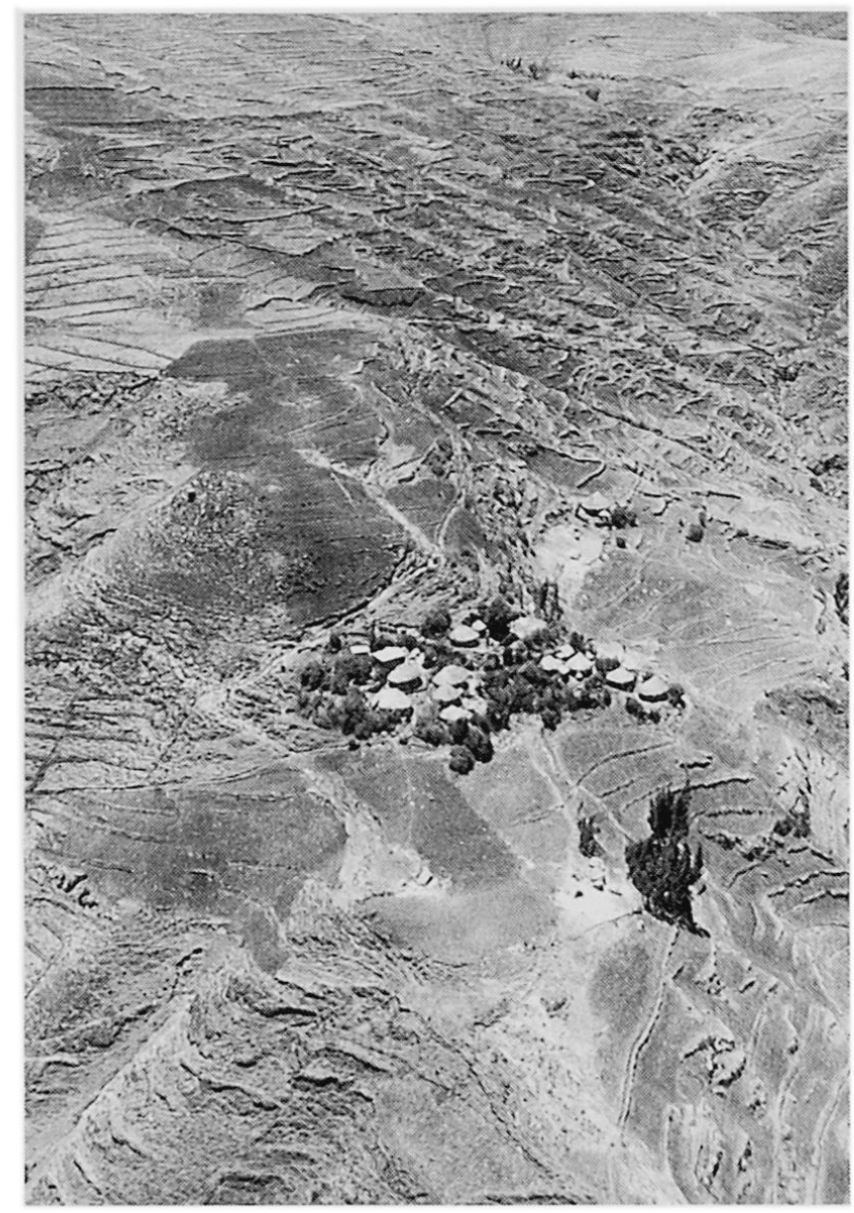

Abb. 2 Der Weiler Astoch (vgl. Abb. 3) in einer äußerst stark geschädigten Kulturlandschaft mit stark reduzierter Bodenproduktivität nach jahrhundertelangem Ackerbau und ungenügendem Bodenschutz.

Luftbild: H. Hurni, Station Andit Tid, April 1985.

Seit der Absetzung Haile Selassies im Jahre 1974 hat die äthiopische Regierung zahlreiche Programme in Gang gesetzt, um mit ländlicher Entwicklung die oben genannten Probleme zu lösen. Neben einer umfassenden Landreform (1975) wurden eigentliche basisdemokratische Bauernvereinigungen (rund $20000 \mathrm{im}$ ganzen Land) aufgebaut und mit der Unterstützung internationaler Organisationen (FAO, World Food Program, UNDP, EG, Bilaterale) Umweltprojekte durchgeführt, die allgemein als erfolgreich beurteilt werden. 
Daneben gab es viele Fehlentwicklungen wie z. B. die einseitige Förderung von Produktionsgenossenschaften und Staatsfarmen, die zwar nur 3\% des landwirtschaftlich genutzten Landes ausmachten, aber über $80 \%$ der staatlichen Förderungsmittel erhielten. Oder die Umsiedlung von 600000 Bauern im Gefolge der Hungersnot von 1984/85, die keine Lösung der Probleme brachte, ebensowenig wie das Dorfprogramm, in welchem die verstreuten Weilersiedlungen zu Dörfern zusammengezogen wurden (HURNI, 1990 b).

Viele Fehlentwicklungen und übereilt geplante Programme hätten mit besseren Kenntnissen lokaler Situationen und regionaler Übersichten vermieden werden können, wenn diese Information verfügbar gewesen und eingebracht worden wäre. Leider aber mangelt es in Äthiopien nicht nur an allen Produkten und Dienstleistungen zur Befriedigung der Grundbedürfnisse, sondern auch an Karten als Grundlage effizienter Entwicklungsplanung. Das Geographische Institut der Uni Bern, das sich seit 1973 in Äthiopien engagiert und seit 1981 mit Unterstützung der «Direktion für Entwicklungszusammenarbeit und humanitäre Hilfe» ein landesweit arbeitendes Forschungsprojekt zur Bodenkonservierung und ländlichen Entwicklung unterhält, beteiligte sich stets auch an der inhaltlichen Gestaltung topographischer und thematischer Karten in verschiedenen Regionen Äthiopiens.

\section{Topographische und thematische Übersichtskarten}

Die erste und bisher einzige topographische Aufnahme des ganzen Landes wurde 1963-1972 mit amerikanischer Hilfe gemacht. 97 Kartenblätter im Maßstab 1: 250000 liefern zuverlässige Informationen zum Relief (100-m-Höhenlinien) und dem damals existierenden Straßennetz. Leider basiert der Waldbestand auf oberflächlicher Interpretation alter Luftbilder, und auf eine genaue Feldverifikation von Ortsnamen und Ortschaften wurde wegen der geringen Zugänglichkeit oft verzichtet. Seit 1971 ist die «Ethiopian Mapping Authority» (EMA) daran, genauere Karten im Maßstab 1:50 000 zu produzieren (20-m-Höhenlinien), die auf deutlich besserer Feldverifikation basieren. Bis 1989 wurden 543 Karten angegangen, die zwar erst $34 \%$ des Landes, aber bereits rund $60 \%$ des Hochlandes decken. Weiter wurden 180 Karten im Maßstab 1:2000 oder $1: 2500$ von Städten produziert. Schließlich stellte die EMA stark generalisierte Karten im Maßstab 1:1000 000 des Landes (nur 500-m-Höhenlinien) sowie eine (relativ ungenaue) Übersichtskarte (1:2000 000) auf Grundlage alter italienischer oder englischer Karten zusammen (EMA, 1990). Letztere Übersichtsmaßstäbe werden zur Zeit in Zusammenarbeit mit unserem Institut auf Grundlage der heute existierenden genaueren Karten neu bearbeitet.

Tab. 1 Thematische Übersichtskarten in Äthiopien mit einer persönlichen Beurteilung ihrer Qualität durch den Autor.

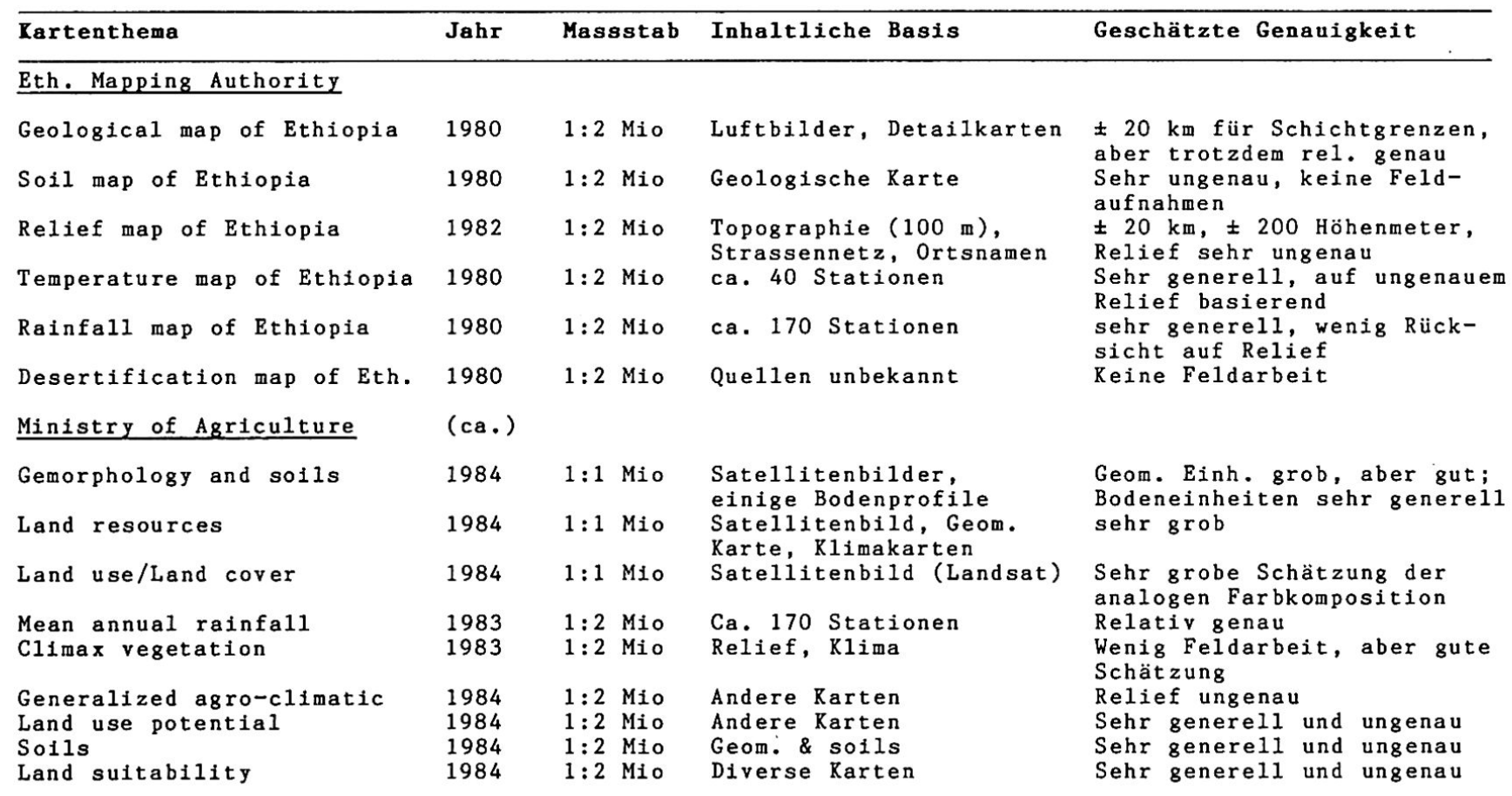


Thematische Karten gibt es in Äthiopien paradoxerweise fast ausschließlich im Übersichtsmaßstab (1:1000 000 oder $1: 2000000$ ), obschon man derartige inhaltliche Information als Generalisierung detaillierter Karten erwarten sollte. Tabelle 1 gibt einen Überblick über die existierenden thematischen Karten in Äthiopien und eine Beurteilung ihrer Qualität. Neben der oben erwähnten systematischen Problematik leiden sämtliche Karten unter der ungenauen topographischen Basis sowie der Tatsache, daß oft inhaltliche Modelle auf das äthiopische Hochland angewendet wurden, deren Genauigkeit nicht mit lokalen Daten überprüft werden konnte.

Aus oben genannten Gründen sind die bisherigen thematischen Karten schlecht geeignet, ländliche Entwicklungsplanung relevant zu fördern. Beispielsweise bestehen über grundlegende Fragen wie
Landnutzung höchst ungenaue Angaben: Der Anteil der ackerbaulich genutzten Fläche schwankt von 8 Millionen bis 18 Millionen Hektaren, je nach Interpretation der Karten!

\section{Karten zur ländlichen Entwicklung}

Das Dilemma der Ungenauigkeit kleinmaßstäbiger Karten und ihrer schlechten Verwendbarkeit für ländliche Entwicklungsplanung kann nur gelöst werden, wenn einerseits genauere, realitätsbezogene Modelle für die jeweils gewünschten größeren Maßstäbe entwickelt werden und andererseits das Problem der Generalisierung und Regionalisierung lokaler Aussagen gelöst werden kann. Von Anbeginn seiner Feldarbeiten in Äthiopien im Jahre 1973 hat sich das Geographische Institut auf derartige

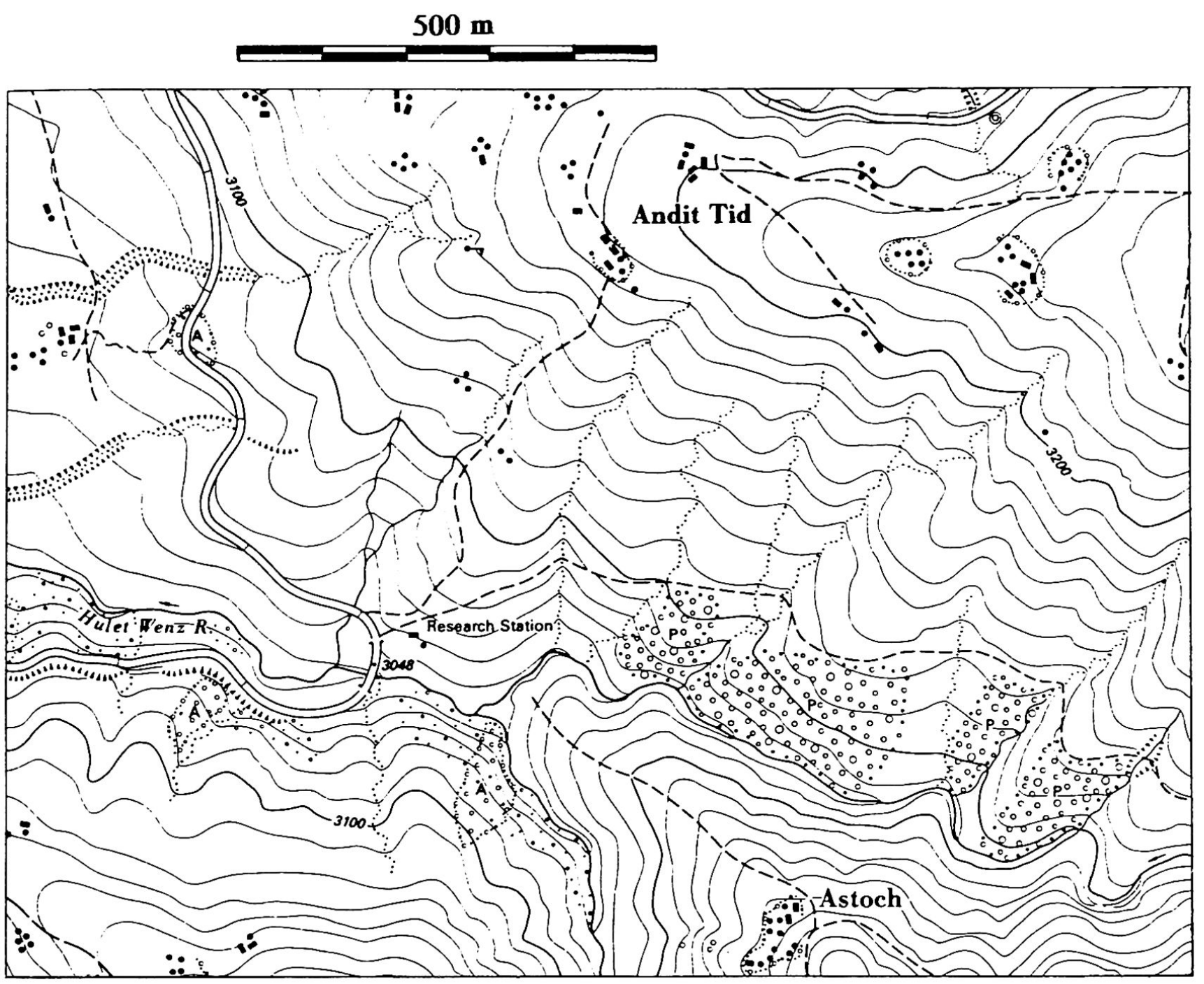

Abb. 3 Ausschnitt der Karte (Originalmaßstab 1 : 10 000) der Forschungsstation Andit Tid/Shewa Region des Bodenkonservierungsprogramms der Universität Bern (950' n. Br., 3943' ö. L.). 


\section{Forschungsprojekt Bodenkonservierung - Äthiopien}

\section{Standardprogramm}

Biologische

Konservierungs-

Versuche

Produktions-

Messungen

Landnutzungs-

Verteilung pro

Anbauperiode

Physikalische

Bodenkonservierung

Erosions-

Testflächen und

Kartierungen

Hydrologie-

Messnetz

Klima-

Stationen

Demographische

Erhebungen

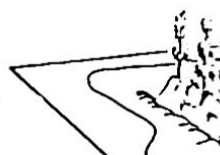

ste

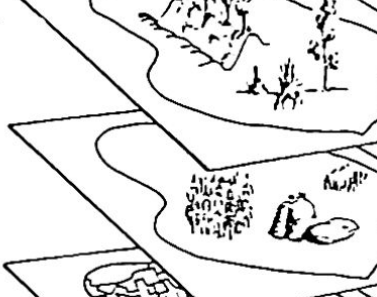

人, Produktivitäts-

(ausgewählte Stationen)
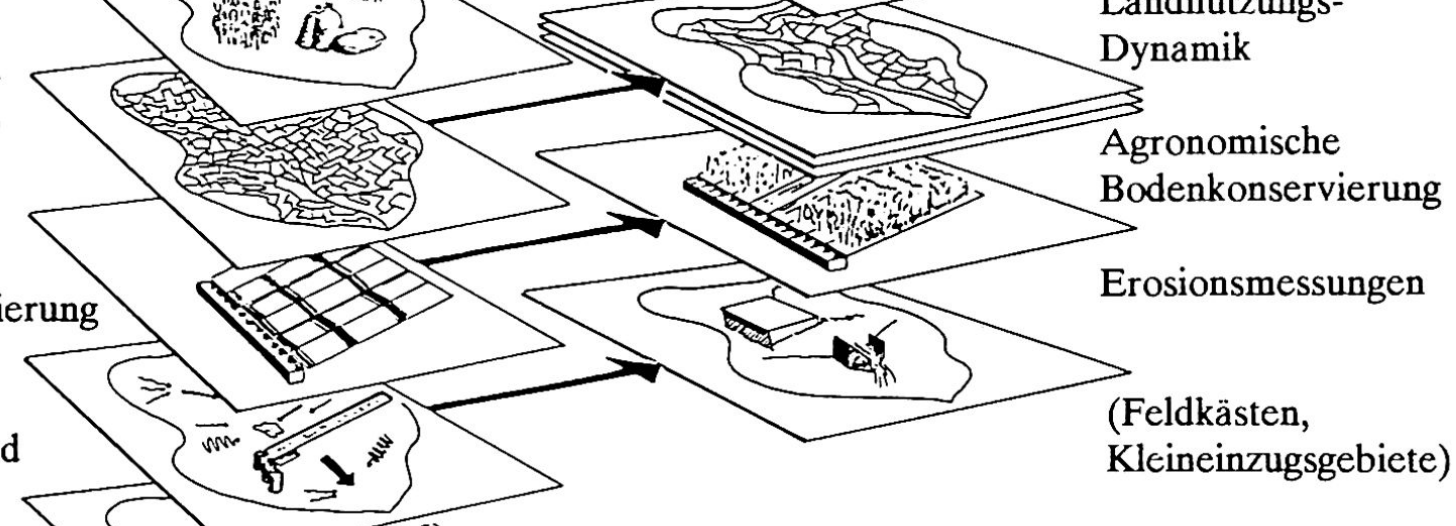

(Feldkästen,

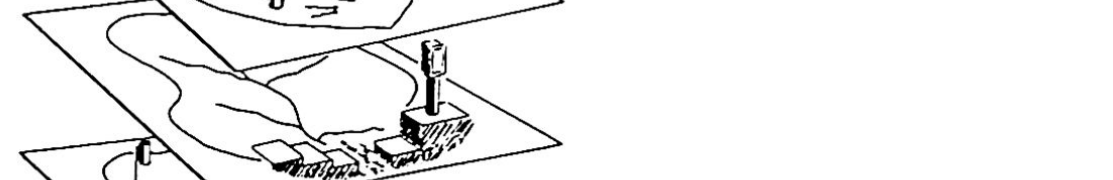

Versuche

Erosionsmessungen

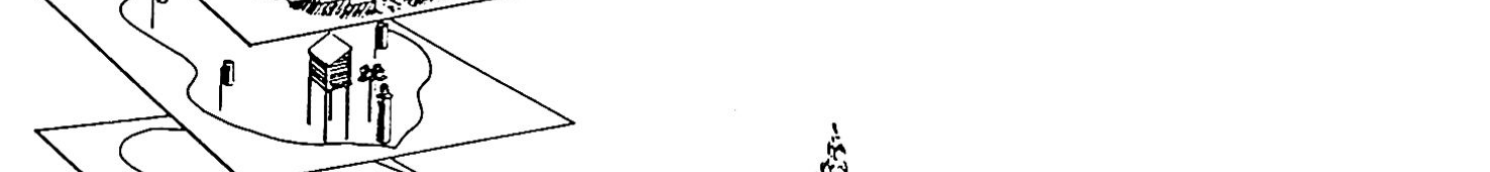

\section{he}
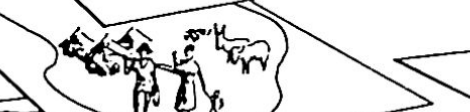

Bodeneinheiten und Degradation

Kleineinzugsgebiete)

Topographie,

Vegetation,

Dörfer, etc.

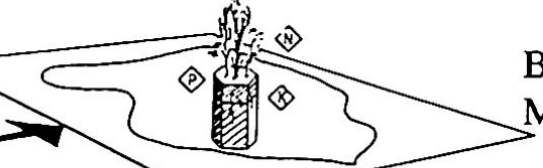

Bodenf ruchtbarkeits-

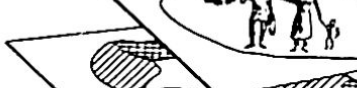

Messungen

Geomorphologie
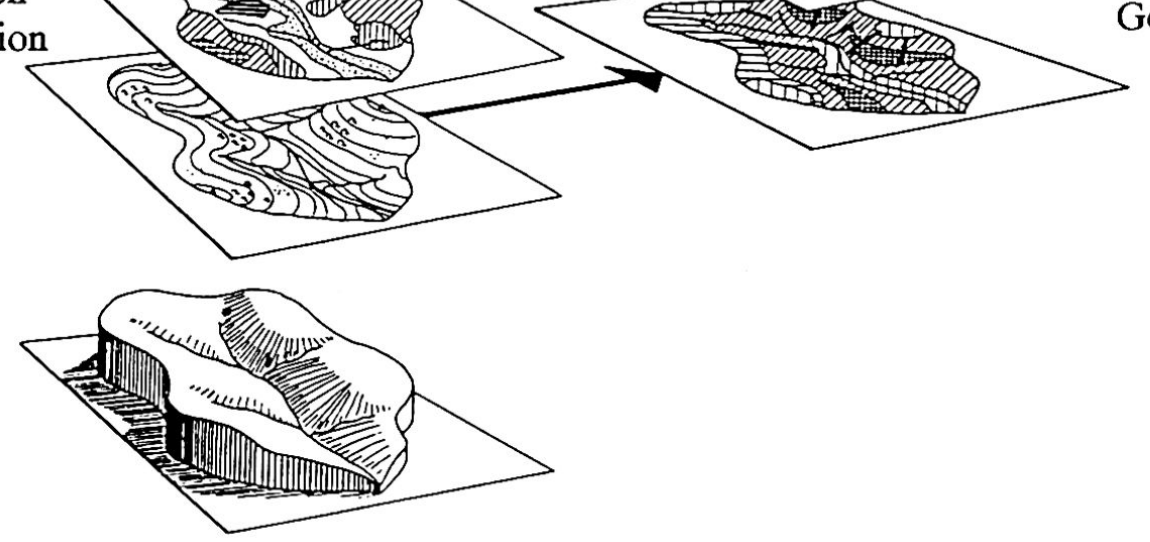

Abb. 4 Räumliche Informationsschichten des Geographischen Informations-Systems (GIS) des Bodenkonservierungsprogramms (Zeichnung: K. Herweg). 
Fragestellungen konzentriert. Im Sammelband «Cartography and its Application for Geographical and Ecological Problems», herausgegeben von B. MESSERLI und K. AERNI (1978), wurden Karten im Maßstab 1:25000 und größer über das Semiengebirge im Norden Äthiopiens zu den Themen Stadtentwicklung, Markt, Landnutzungsentwicklung und Bodenerosion publiziert, die bereits wertvolle Grundlagen für Entwicklungsprogramme lieferten. Beispielsweise konnte der bekannte Semien-Nationalpark detailliert geplant werden (HURNI. 1986), es wurde erstmals eindrücklich demonstriert, wie sich die Bevölkerungsentwicklung auf die Ausdehnung des Kulturlandes auswirkte, oder es zeigte sich, daß die Bodenerosionsschäden das Resultat jahrhundertelanger Nutzung ohne Bodenkonservierung sind.

Letzteres Thema wird seit 1981 in einem landesweiten Netz von sieben in verschiedenen agro-ökologischen Zonen gelegenen Forschungsstationen des «Soil Conservation Research Project» des Instituts in Zusammenarbeit mit dem äthiopischen Landwirtschaftsministerium intensiviert weiterverfolgt (HURNI, 1982).

Jedes der 1-7 km² großen Einzugsgebiete wurde im Maßstab 1:5000 neu kartiert und im Maßstab $1: 10000$ publiziert (Abb. 3). Diese topographischen Grundlagen bildeten die Basis für Bodenkarten (BONO and SEILER, 1983, 1984; WEIGEL, 1986; KEFENI KEJELA, in Vorbereitung) und dienen auch für die regelmäßige Kartierung der Landnutzung jeder Anbauperiode seit 1981. Das ganze Forschungsprogramm basiert auf einem geographischen Informationssystem, wie es in Abb. 4 dargestellt wird.

Die Analyse der langjährigen Erhebungen erlaubt eine Vielzahl von Rückschlüssen zur Dynamik der Bodenerosion (vgl. BELAY TEGENE, in Vorbereitung), der Landnutzung, der Produktion oder der Wirksamkeit von Bodenkonservierung. Abb. 5 zeigt beispielsweise das Resultat der raum-zeitlichen Anbau- und Produktionsstudie in der Station Hunde Lafto/Harerge mit Daten, die von B. SCHÜPBACH (1988) zusammengestellt wurden. Aus der Gesamtproduktion ist einerseits 1984 der massive Produktionseinbruch wegen Dürre klar interpretierbar und andererseits ein leichter Trend zur absoluten $\mathrm{Ab}$ nahme der Produktion im Einzugsgebiet von 19821986 feststellbar, dessen Auswirkungen wegen der Bevölkerungszunahme noch relativ verstärkt werden.

Derartige Trends lassen sich aber nicht nur für einzelne Forschungsstationen feststellen, sondern mit bestimmten Modellen und unter bestimmten Annahmen auch im landesweiten Maßstab (Abb.6, HURNI, 1990 a). Hier zeigt sich deutlich, daß bei der gegenwärtigen Entwicklungsdynamik vor allem wegen der Bevölkerungszunahme in den nächsten 50 Jahren die heute noch vorhandenen Ressourcen klar ungenügend sein werden, mit massiven natio-

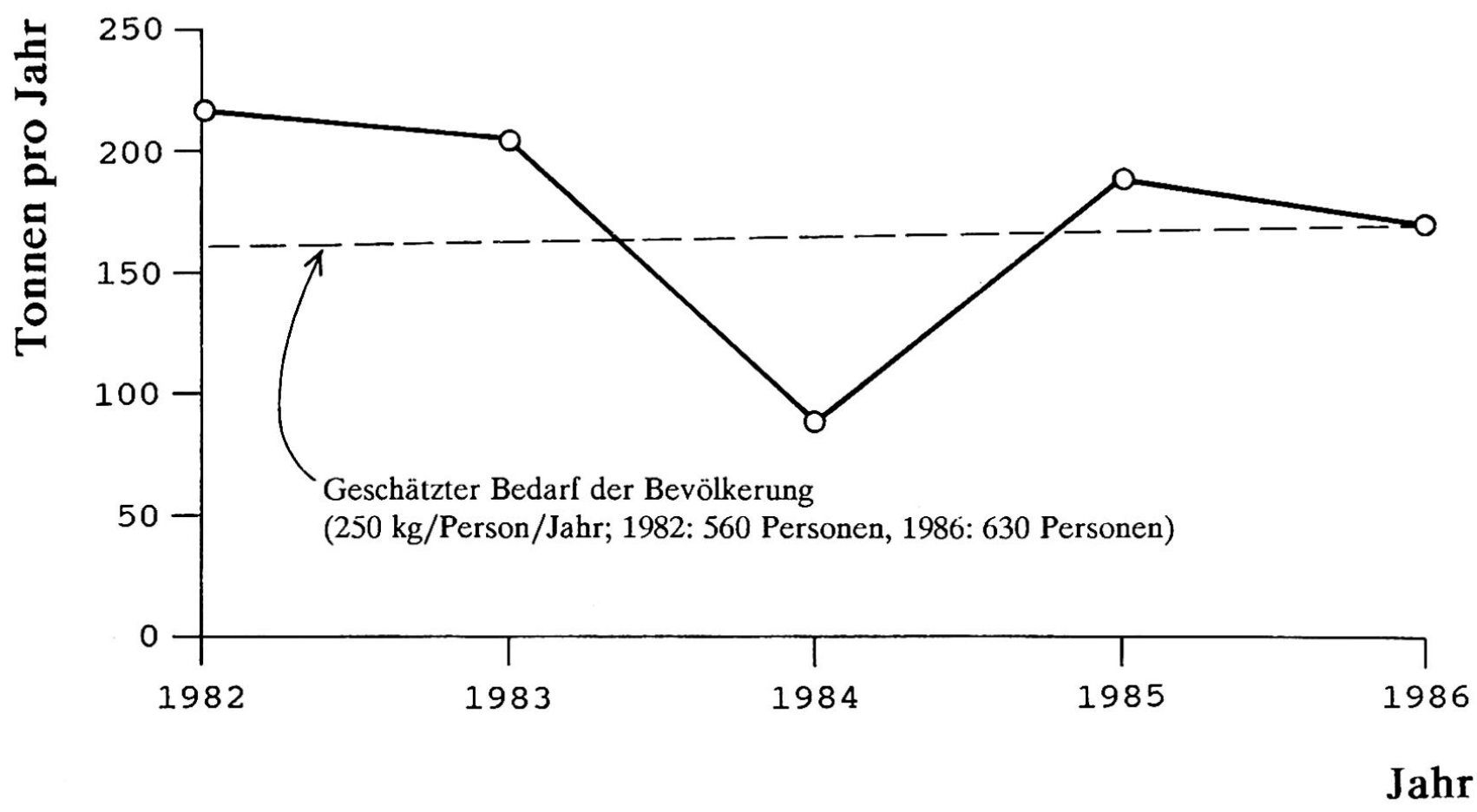

Abb. 5 Nahrungsmittel-Gesamtproduktion des 2,34 km² großen Einzugsgebietes Hunde Lafto des Bodenkonservierungsprogramms in der Harerge-Region von 1982 bis 1986 (Zusammenstellung durch B. Schüpbach, 1989). 


\section{Szenarium "Heutige Entwicklungstrends"}

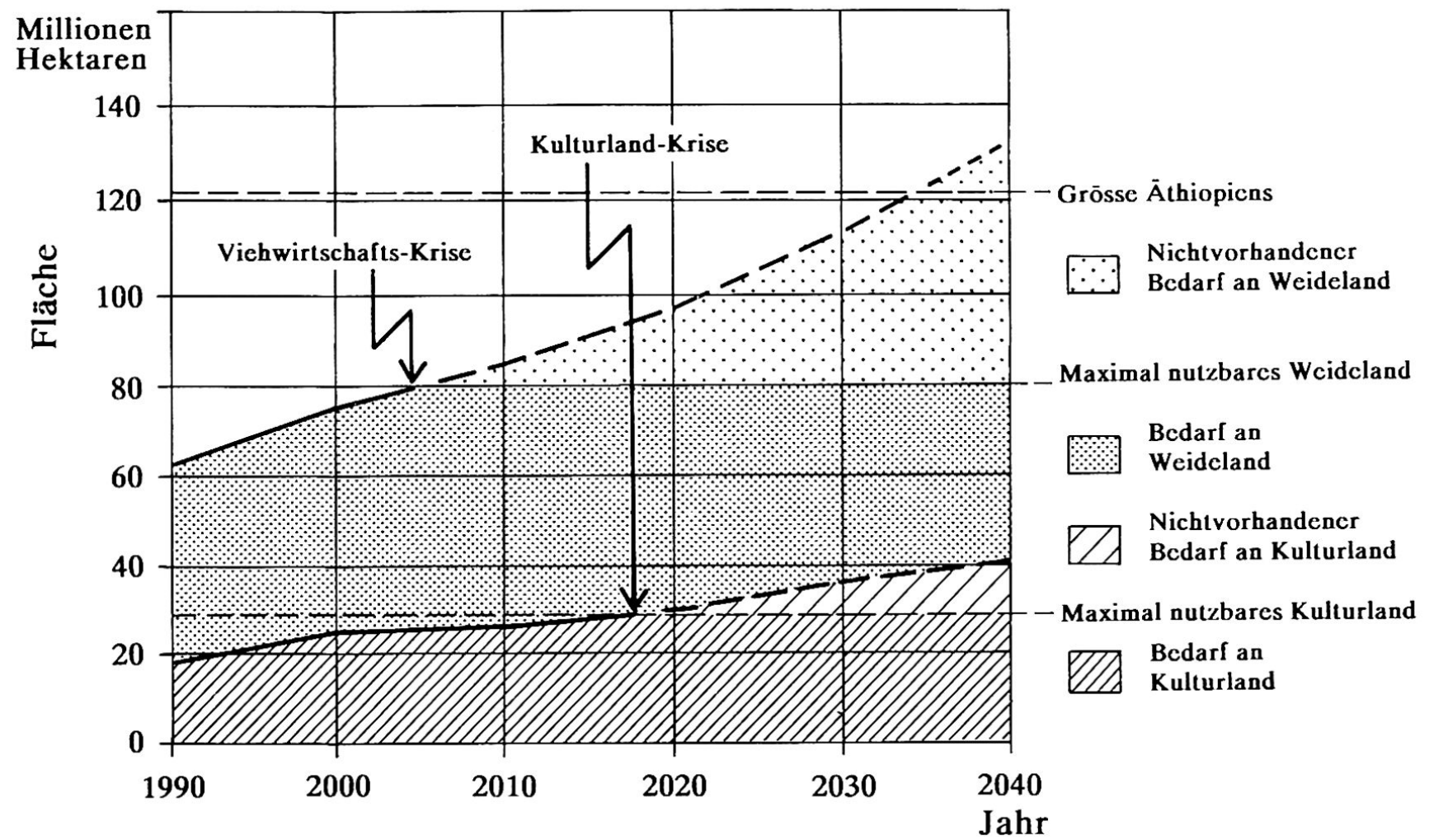

Abb. 6 Hypothetischer Landbedarf der äthiopischen Landwirtschaft bei heutigen Entwicklungstrends und Degradation in den nächsten 50 Jahren (Hurni, 1990 a).

nalen Krisen in der Viehwirtschaft wegen Verknappung des Weidelandes schon in den nächsten 15 Jahren, und einer Krise des Kulturlandes in etwa 25 Jahren.

\section{Ausblick}

Derartige globale Ressourcennutzungsmodelle wie oben beschrieben können erst ernst genommen werden, wenn sie auf soliden, großmaßstäbig erhobenen Daten und Prozessen in den diversen ökologischen und kulturellen Zonen des riesigen Hochlandes basieren. Obschon in Ansätzen vorhanden, sind die existierenden Daten, Modelle und Resultate viel zu spärlich, um statistisch signifikante Extrapolationen zuzulassen.

Großmaßstäbige Beiträge wie diejenigen des «Soil Conservation Research Project» in Äthiopien sind zwar konzeptionell richtig angelegt, kommen aber angesichts der drohenden Katastrophen relativ spät. Trotzdem müssen derartige Anstrengungen um ein Vielfaches verstärkt werden. Gleichzeitig können auch kleinmaßstäbige Beiträge deutlich verbessert werden, indem beispielsweise die von Satelliten aufgenommene Information vermehrt mitein- bezogen wird. Allgemein liegt in der kartographischen und thematischen Informationsbeschaffung in Äthiopien ein grosses Potential zur Verbesserung der Situation, denn räumlich differenzierte und zeitlich dynamische Aussagen sind für eine verbesserte Entwicklungspolitik dieses riesigen Landes von entscheidender Bedeutung.

\section{Zusammenfassung}

Äthiopiens Probleme der Ressourcenübernutzung, der Unterentwicklung, der Anfälligkeit für Hungersnöte und der regionalen Konflikte sind auf seine gebirgige Lage, seine agroökologische Vielfalt, sein kulturgeschichtliches Alter und die Einflüsse von außen zurückzuführen. Viele gutgemeinte Entwicklungsbemühungen scheitern zudem an mangelnder räumlicher Information als Planungsgrundlage. Die nationalen und internationalen Bemühungen zur Beseitigung dieses Engpasses, mit besonderer Berücksichtigung topographischer und thematischer Karten als Grundlage ländlicher Entwicklung, werden in diesem Artikel angesprochen. 


\section{Literaturverzeichnis}

BELAY TEGENE, in prep.: Erosion: Its effects on properties and producitivity of a Nitosol in Southern Ethiopia, and some techniques of its control (working title). PhD thesis, Berne University, Switzerland.

BONO, R. and SEILER, W. (1983): The soils of the Suke-Harerge Research Unit (Ethiopia). Classification, morphology and ecology, with map 1:5000. Research Report 2, with supplement. Soil Conservation Research Project, Addis Abeba, $95 \mathrm{pp}$.

BONO, R. and SEILER, W. (1984): The soils of the Andit Tid Research Unit (Ethiopia). Classification, morphology and ecology, with soil map 1:5000. Research Report 3, with supplement. Soil Conservation Research Project, Addis Abeba, 80 pp.

CONSTABLE, M. (1985): EHRS Summary (Draft). Ethiopian Highlands Reclamation Study, UTF/ETH/037/ETH Working Paper 24. FAO, Rome, 34 pp.

EMA (1990): Map Catalogue. Ethiopian Mapping Authority Addis Abeba, 32 pp.

HURNI, H. (1982): Inception Report, Soil Conservation Research Project, Vol. 1, June 1982. University of Berne, 51 pp.

HURNI, H. (1986): Management Plan, Simen Mountains National Park and Surrounding Rural Area, Ethiopia. UNESCO World Heritage Committee, $122 \mathrm{pp}$.
HURNI, H. (1990 a): Land degradation, famine, and land resource scenarios in Ethiopia. World Soil Erosion and Conservation. D. Pimentel (Edt.). IUCN, Gland, Switzerland (im Druck).

HURNI, H. (1990 b): Agrarentwicklung und Umweltprobleme in Äthiopien. Kapitel im Buch von V. Mathies und S. Brüne (Hrsg.) in der Instituts-Reihe des Afrika-Instituts, Hamburg (im Druck).

KEFENI KEJELA, in prep.: Comparative evaluation of the impact of different soil conservation measures on soil productivity and agricultural production in Anjeni Area, West Gojam, Ethiopia (working title). PhD thesis, Berne University, Switzerland.

MESFIN WOLDE-MARIAM (1984): Rural vulnerability to famine in Ethiopia: 1958-1977. Vikas Publishing House, Sahibadad, India, $191 \mathrm{pp}$.

MESSERLI, B. and AERNI, K. (edts), (1978): Cartography and its application for geographical and ecological problems. Simen Mountains-Ethiopia, Vol. 1, Geographica Bernensia G8, Bern, 102 pp.

SCHÜPBACH, B. (1988): Veränderungen in Landnutzung Nahrungsmittel- und Biomassenproduktion zwischen 1982 und 1986 und ihre Auswirkungen auf die Bevölkerung im Agucho-Tal, Harerge/Äthiopien. Universität Bern, $72 \mathrm{~S}$.

WEIGEL, G. (1986): The Soils of the Maybar/Wello Area. Their potential and constraints for agricultural development. A case study in the Ethiopian Highlands. Geographica Bernensia Vol. A4, Berne, $173 \mathrm{pp}$. 